\title{
UNITARY HARMONIC NUMBERS
}

\section{PETER HAGIS, JR. AND GRAHAM LORD}

ABSTRACT. If $d^{*}(n)$ and $\sigma^{*}(n)$ denote the number and sum, respectively, of the unitary divisors of the natural number $n$ then the harmonic mean of the unitary divisors of $n$ is given by $H^{*}(n)=n d^{*}(n) / \sigma^{*}(n)$. Here we investigate the properties of $H^{*}(n)$, and, in particular, study those numbers $n$ for which $H^{*}(n)$ is an integer.

1. Introduction. Let $d(n)$ and $\sigma(n)$ denote, respectively, the number and sum of the positive divisors of the natural number $n$. Ore [6] showed that the harmonic mean of the positive divisors of $n$ is given by $H(n)=$ $n d(n) / \sigma(n)$, and several papers (see [1], [5], [6], [7]) have been devoted to the study of $H(n)$. In particular the set of numbers $S$ for which $H(n)$ is an integer has attracted the attention of number theorists, since the set of perfect numbers is a subset of $S$. The elements of $S$ are called harmonic numbers by Pomerance [7]. This paper is devoted to a study of the unitary analogue of $H(n)$. We recall that the positive integer $d$ is said to be a unitary divisor of $n$ if $d \mid n$ and $(d, n / d)=1$. It is easy to verify that if the canonical prime decomposition of $n$ is given by

$$
n=p_{1}^{a_{1}} p_{2}^{\alpha_{2}} \cdots p_{k}^{\alpha_{k}}
$$

and $d^{*}(n)$ and $\sigma^{*}(n)$ denote the number and sum, respectively, of the unitary divisors of $n$ then

$$
d^{*}(n)=2^{k} ; \quad \sigma^{*}(n)=\left(p_{1}^{a_{1}}+1\right)\left(p_{2}^{a_{2}}+1\right) \cdots\left(p_{k}^{\alpha_{k}}+1\right) .
$$

It is also easy to show that the unitary harmonic mean (the harmonic mean of the unitary divisors) of $n$ is given by

$$
H^{*}(n)=n d^{*}(n) / \sigma^{*}(n)=\prod_{i=1}^{k} 2 p_{i}^{a_{i}} /\left(p_{i}^{a_{i}}+1\right) \text {. }
$$

We shall say that $n$ is a unitary barmonic number if $H^{*}(n)$ is an integer,

Presented to the Society, January 25, 1975; received by the editors April 29, 1974. AMS (MOS) subject classifications (1970). Primary 10A20. 
and shall denote by $U H$ the set of these numbers. A computer search (which required approximately 2.5 hours of CDC 6400 time at the Temple University Computer Center) was made for the elements of $U H$ in the interval $\left[1,10^{6}\right]$, and 45 such numbers were found. These are given in Table I at the end of this paper.

Subbarao and Warren [10] have defined $n$ to be a unitary perfect number if $\sigma^{*}(n)=2 n$. Five such numbers are presently known [9]. Since $d^{*}(n)$ is even the following result is immediate from (3).

Proposition 1. The set of unitary perfect numbers is a subset of $U H$.

2. Some elementary results concerning $H^{*}(n)$ and $U H$. We now establish some facts which will be of use in the sequel and which, in certain cases, are of some interest in themselves. $n$ will always denote a natural number with prime decomposition as given in $(1) ; p, q, r$ with or without subscripts will always denote primes.

Lemma 1. $2^{k+1} /(k+2) \leq H^{*}(n)<2^{k}$ with equality on the left if and only if $n=2$ or 6 .

Proof. Since $x /(x+1)$ is monotonic increasing and bounded by 1 for positive $x$, it follows from (3) that

$$
2^{k}>H^{*}(n) \geq 2^{k}(2 / 3)(3 / 4)(4 / 5) \cdots((k+1) /(k+2))=2^{k+1} /(k+2) .
$$

Lemma 2. If $p^{\alpha} \| n$, then $p^{\alpha} \geq H^{*}(n) /\left(2^{k}-H^{*}(n)\right)$ with equality if and only if $k=1$.

Proof. From (3), $H^{*}(n) \leq 2^{k} p^{a} /\left(p^{a}+1\right)$.

Lemma 3. If $p^{a}\left\{r^{c}\right\}$ is the minimum $\{$ maximum $\}$ prime power divisor of $n$ in (1) then

$$
p^{a} \leq k H^{*}(n) /\left(2^{k}-H^{*}(n)\right) \quad\left\{r^{c} \geq\left((k-1) 2^{k}+H^{*}(n)\right) /\left(2^{k}-H^{*}(n)\right)\right\}
$$

with equality if and only if $k=1$ or $n=p^{a} q^{b} r^{c}$ where $q^{b}=p^{a}+1$ (so that $2 \mid n)$ and $r^{c}=p^{a}+2$ or $c=0$ \{wbere $q^{b}=r^{c}-1$ (so that $\left.2 \mid n\right)$ and $p^{a}=$ $r^{c}-2$ or $\left.a=0\right\}$.

Proof.

$$
\begin{aligned}
H^{*}(n) & \geq 2^{k}\left\{p^{a} /\left(p^{a}+1\right)\right\}\left\{\left(p^{a}+1\right) /\left(p^{a}+2\right)\right\} \cdots\left\{\left(p^{a}+k-1\right) /\left(p^{a}+k\right)\right\} \\
& =2^{k} p^{a} /\left(p^{a}+k\right),
\end{aligned}
$$


and

$$
\begin{aligned}
H^{*}(n) & \leq 2^{k}\left\{\left(r^{c}-k+1\right) /\left(r^{c}-k+2\right)\right\}\left\{\left(r^{c}-k+2\right) /\left(r^{c}-k+3\right)\right\} \cdots\left\{r^{c} /\left(r^{c}+1\right)\right\} \\
& =2^{k}\left(r^{c}-k+1\right) /\left(r^{c}+1\right)
\end{aligned}
$$

with equality only in the specified "exceptional" cases.

We turn now to some results concerning $U H$. The following proposition was proved by Ore [6, p. 617] for harmonic numbers and holds for elements of $U H$ since $H(n)=H^{*}(n)$ if and only if $n$ is square-free.

Proposition 2. If $n$ is square-free and $n \neq 6$, then $n$ is not a unitary barmonic number.

Since $2 \mid\left(p^{\alpha}+1\right)$ if $p$ is odd, and since $4 \mid\left(p^{\alpha}+1\right)$ if $p=4 j+3$ and $a$ is odd, our next two results follow immediately from (3).

Proposition 3. If $n$ is odd and $n \in U H$, then $H^{*}(n)$ is odd.

Proposition 4. If $n$ is odd, $n \in U H, p^{a} \| n$, and $p=4 j+3$ then $a$ is even.

From (2) and (3) it is immediate that $H^{*}(n)$ is a multiplicative function. Therefore, if $(n, m)=1$ then $H^{*}(n m)=H^{*}(n) \cdot m d^{*}(m) / \dot{\sigma}^{*}(m)$ from which we easily deduce the following result.

Proposition 5. If $n \in U H,(p, n)=1$, and $\left(p^{a}+1\right) \mid 2 H^{*}(n)$, then $p^{a} n \in U H$.

For example, since $40950 \epsilon U H$ and $30=2 H^{*}(40950)$ we see that 29. $40950 \in U H$ also.

\section{Two cardinality theorems.}

Theorem 1. If $S_{c}$ is the set of natural numbers $n$ such that $H^{*}(n)=c$, then $S_{c}$ is finite (or empty) for every real number $c$.

Proof. Our proof is based on an idea due to Shapiro [8]. Since $2^{k+1} /(k+2) \geq k$ we note first that if $H^{*}(n)=c$ then Lemma 1 implies that the number of prime factors of $n$ is bounded (by $c$ ). Now assume that $S_{c}$ is infinite. Then $S_{c}$ must contain an infinite subset, say $S_{c m}$, each of whose elements has exactly $m$ prime factors. It is not difficult to see that an infinite sequence $n_{1}, n_{2}, \cdots$ of distinct integers exists with the following properties: 
(i) $n_{i} \in S_{c m}$ so that $H^{*}\left(n_{i}\right)=c$ for $i=1,2, \cdots$;

$$
n_{i}=p_{1}^{a_{1}} \cdots p_{s-1}^{a_{s-1}} p_{i s}^{a_{i s}} \cdots p_{i m}^{a_{i m}}=P \prod_{j=s}^{m} p_{i j}^{a_{i j}}
$$

where

$$
p_{1}^{a_{1}}<\cdots<p_{s-1}^{a_{s}-1}<p_{i s}^{a_{i s}}<\cdots<p_{i m}^{a_{i m}} \text { for } i=1,2, \cdots
$$

( $P$ may be empty, but $s-1 \neq m$.)

(iii) $p_{i j}^{\alpha_{i j}} \rightarrow \infty$ as $i \rightarrow \infty$ for $j=s, \cdots, m$.

(That is, each $n_{i}$ is composed of a fixed, constant block of prime powers and a variable block of prime powers arranged monotonically within the block and such that each component of this variable block goes to infinity with i.)

From (i) and (ii) we see that

$$
\frac{c}{H^{*}(P)}=\prod_{j=s}^{m} H^{*}\left(p_{i j}^{a_{i j}}\right)<2^{m+1-s}
$$

so that there exists a fixed positive number $v$ such that $\Pi_{j=s}^{m} H^{*}\left(p_{i j}^{\alpha_{i j}}\right)=$ $2^{m+1-s}-v$ for $i=1,2,3, \cdots$. But from (iii) it follows that $H^{*}\left(p_{i j}^{a_{i j}}\right) \rightarrow 2$ as $i \rightarrow \infty$ for $s \leq j \leq m$. Therefore, for "large" $i$, $\Pi_{j=s}^{m} H^{*}\left(p_{i j}^{\alpha_{i j}}\right)>2^{m+1-s}-v$. This contradiction completes the proof.

Since there are only finitely many integers between $2^{k+1} /(k+2)$ and $2^{k}$ the following theorem follows from Lemma 1 and Theorem 1 .

Theorem 2. There exist at most finitely many unitary barmonic numbers with a specified number of distinct prime factors.

From Proposition 1 we have the following corollary which was first proved by Subbarao and Warren [10].

Corollary 2.1. There are at most a finite number of unitary perfect numbers with a specified number of prime factors.

4. Elements of $U H$ with a specified number of prime factors. Let $T_{k}$ denote the set of unitary harmonic numbers which have exactly $k$ prime factors. In connection with Theorem 2 it is perhaps of some interest to identify the elements of $T_{k}$ for a few selected values of $k$.

Proposition 6. $T_{1}$ is empty.

Proof. $H^{*}\left(p^{\alpha}\right)=2 p^{\alpha} /\left(p^{\alpha}+1\right)$, and it is easy to see that $\left(p^{a}+1\right) \not 2 p^{\alpha}$.

Proposition 7. $T_{2}=\{6,45\}$. 
Proof. If $n \in T_{2}$ then, from Lemma $1, H^{*}(n)=2$ or 3 . If $H^{*}(n)=2$ then from Leinma 3, $2 \| n$ and consequently $n=6$. If $H^{*}(n)=3$ then from (3) and Lemma $2,3^{b} \| n$ where $b>1$. From Lemmas 2 and 3 either $2^{2} \| n$ or $5 \| n$. If $n=2^{2} 3^{b}$ then $5\left(3^{b}+1\right)=16 \cdot 3^{b-1}$ which is impossible. If $n=$ $5 \cdot 3^{b}$ then $3=H^{*}(n) \geq H^{*}\left(5 \cdot 3^{2}\right)=3$ so that $n=45$.

Proposition 8. $T_{3}=\{60,90,1512,15925,55125\}$.

Proof. From Lemma $1 H^{*}(n)=4,5,6$ or 7 if $n \in T_{3}$. We consider these possibilities separately. $p^{a}$ will always denote the minimal prime power in (1). It can be bounded by using Lemmas 2 and 3. We shall also rely heavily on the fact that $x /(x+1)$ is monotonic increasing.

Case I. $H^{*}(n)=4$. Then $p^{a}=2$ or 3 , and from Proposition $3 n$ is even. If $p^{a}=2$, then, since $n$ is not square-free, $4=H^{*}(n) \geq H^{*}\left(2 \cdot 3^{2} \cdot 5\right)$ =4. If $p^{a}=3$ then $4=H^{*}(n) \geq H^{*}\left(2^{2} \cdot 3 \cdot 5\right)=4$. Therefore, $n=60$ or 90 .

Case II. $H^{*}(n)=5$. $p^{a}=2,3$ or 4 , and from (3) $5 \mid n$. If $p^{a}=2$ then $n=2 \cdot 3^{b} 5^{c}$, and from (3): $\left(3^{b-1}-5\right)\left(5^{c-1}-3\right)=16$ which is impossible. If $p^{a}=3$ then from Proposition $4 n$ is even and $n=2^{b} 3 \cdot 5^{c}$. It follows that $\left(2^{b}-5\right)\left(5^{c-1}-1\right)=6$ which is impossible. If $p^{a}=4$ then $n=4 \cdot 5^{b} q^{c}$ and $5=H^{*}(n) \geq H^{*}\left(20 q^{c}\right)=16 q^{c} / 3\left(q^{c}+1\right)$ which implies that $q^{c}=7,9,11$ or 13. Each of these possibilities leads to a contradiction.

Case III. $H^{*}(n)=6$. Then $n$ is even, $3 \mid n$, and $p^{a}=4,5,7$ or 8 . If $p^{a}=4$ then $n=4 \cdot 3^{b} \cdot 5^{c}$; if $p^{a}=5$ then $n=2^{b} 3^{c} 5$; if $p^{a}=8$ then $n=$ $8 \cdot 3^{b} \cdot q^{c}$ where $q^{c} \geq 11$. From (3): $\left(3^{b-1}-5\right)\left(5^{c-1}-3\right)=16$; or $\left(2^{b}-9\right)\left(3^{c-2}-1\right)=10$; or $\left(5 \cdot 3^{b-3}-1\right)\left(5 q^{c}-27\right)=32$. None of these is possible. If $p^{a}=7$ then $n=2^{b} 3^{c} 7$, and $\left(2^{b-1}-3\right)\left(3^{c-1}-2\right)=7$. Therefore, $b=c=3$ and $n=1512$.

Case IV. $H^{*}(n)=7$. Then $7 \mid n$ and $p^{a}=8,9,11,13,16,17$ or 19. Assume first that $2 \mid n$. Then, if $p^{a}=9,11,17$ or $19, n$ has four prime factors (from (3)). If $p^{a}=13$ then $H^{*}(n) \geq H^{*}\left(2^{5} 7^{3} 13\right)>7$; if $p^{a}=16$ then $H^{*}(n) \geq H^{*}\left(16 \cdot 7^{3} 17\right)>7$; if $p^{a}=8$ then $n=8 \cdot 3^{b} 7^{c}$, and $\left(3^{b-2}-7\right) \cdot$ $\left(7^{c-1}-9\right)=64$ which is impossible. Now assume that $n$ is odd. Then, using Proposition $4,7^{2} \mid n$; and $p^{a}=9,13$ or 17 . If $p^{a}=17$ then $H^{*}(n) \geq$ $H^{*}\left(17 \cdot 3^{4} \cdot 7^{2}\right)>7$. If $p^{a}=9$ then $n=9 \cdot 5^{b} 7^{c}$, and $\left(5^{b-1}-7\right)\left(7^{c-1}-5\right)=$ 36. Therefore, $b=3, c=2$ and $n=55125$. If $p^{a}=13$ then $n=13 \cdot 7^{b} q^{c}$. If $b \geq 4$ then, from (3), $7^{2} \mid\left(q^{c}+1\right)$ so that $q^{c} \geq 97$. But $H^{*}\left(13 \cdot 7^{4} 97\right)>7$. Therefore, $b=2$ and it then follows that $n=13 \cdot 7^{2} \cdot 5^{2}=15925$.

5. The distribution of the unitary harmonic numbers. For each positive real number $x$ we shall denote by $A(x)$ the number of integers $n$ such that 
$n \leq x$ and $n \in U H$. This section is devoted to a proof of

Theorem 3. For any $\epsilon>0, A(x)<2.2 x^{1 / 2} 2^{(1+\epsilon) \log x / \log \log x}$ for "large" $x$.

Proof. We use an argument of Kanold [5]. A powerful number is a positive integer $m$ with the property that if $p \mid m$ then $p^{2} \mid m$. It is obvious that every positive integer can be written uniquely in the form $N_{P} N_{F}$ where $\left(N_{P}, N_{F}\right)=1, N_{P}$ is powerful (or 1 ) and $N_{F}$ is square-free. If $P(x)$ is the number of powerful numbers not exceeding $x$ it is proved in [2] that $P(x) \sim$ $c x^{1 / 2}$ where $c=\zeta(3 / 2) / \zeta(3)=2.173 \cdots$. It follows that $P(x)<2.2 x^{1 / 2}$ for large $x$.

If $N_{P}$ is a (fixed) powerful number let $g\left(N_{P}, x\right)$ denote the number of square-free numbers $N_{F}$ such that $\left(N_{P}, N_{F}\right)=1, N_{P} N_{F} \leq x$, and $N_{P} N_{F} \epsilon$ $U H$. If $G(x)=\max \left\{g\left(N_{P}, x\right)\right\}$ for $N_{P} \leq x$ it follows that

$$
A(x)<2.2 x^{1 / 2} G(x) \text { for large } x
$$

We now investigate the magnitude of $G(x)$. Let $N_{P}$ be a powerful number for which square-free numbers $m_{1}, m_{2}, \cdots, m_{G(x)}$ exist such that $\left(N_{P}, m_{i}\right)=1, N_{P} m_{i} \leq x$ and $N_{P} m_{i} \in U H$ for $i=1,2, \cdots, G(x)$. Then $H^{*}\left(N_{P} m_{i}\right)=H^{*}\left(N_{P}\right) H^{*}\left(m_{i}\right)=Z_{i}$ where $Z_{i}$ is an integer for $i=1, \cdots, G(x)$. If $Z_{i}=Z_{j}$ where $i \neq j$, and $\left(m_{i}, m_{j}\right)=d$ then, of course, $H^{*}\left(M_{i}\right)=H^{*}\left(M_{j}\right)$ where $M_{i}=m_{i} / d$ and $M_{j}=m_{j} / d$. If $M_{i}=p_{1} \cdots p_{s}$ (or 1 ) and $M_{j}=$ $q_{1} \cdots q_{t}$ (where $p_{1}<\cdots<p_{s}, q_{1}<\cdots<q_{t}$ and $p_{u} \neq q_{v}$ ) then from (3):

$$
2^{s} p_{1} \cdots p_{s}\left(1+q_{1}\right) \cdots\left(1+q_{t}\right)=2^{t} q_{1} \cdots q_{t}\left(1+p_{1}\right) \cdots\left(1+p_{s}\right) .
$$

It is not difficult to see that $p_{s} \leq 3$ and $q_{t} \leq 3$ so that (assuming $M_{i}<M_{j}$ ) $M_{j}=6, M_{i}=1 ; M_{j}=3, M_{i}=1$ or $2 ; M_{j}=2, M_{i}=1$ are the only logical possibilities. Since none of these satisfies (5) we conclude that $Z_{i} \neq Z_{j}$ unless $i=j$. Therefore, without loss of generality, $Z_{1}<Z_{2}<\cdots<Z_{G(x)}$ so that $G(x) \leq Z_{G(x)}=H^{*}\left(N_{P} m_{G(x)}\right)<2^{k}$, where $k$ is the number of prime factors in $N_{P} m_{G(x)}$. If $N=2 \cdot 3 \cdot 5 \cdots p_{K}$ is the "longest prime product" not exceeding $x$ then $k \leq K$. Since $K \sim \log N / \log \log N(\operatorname{see}[4, \$ 22.10]$ ) it follows that if $\epsilon>0$ then

$$
G(x)<2^{(1+\epsilon) \log x / \log \log x} \text { for large } x .
$$

Our theorem follows from (4) and (6).

Remark. It follows easily from Theorem 3 that $U H$ has zero density. That the set of unitary perfect numbers has density zero was first shown by Subbarao [3, p. 1117]. 
Table 1. The unitary harmonic numbers in $\left[1,10^{6}\right]$.

\begin{tabular}{|c|c|c|c|c|c|}
\hline$n$ & $H^{*}(n)$ & $n$ & $H^{*}(n)$ & $n$ & $H^{*}(n)$ \\
\hline 1 & 1 & 27300 & 15 & 232470 & 15 \\
\hline 6 & 2 & 31500 & 10 & 257040 & 20 \\
\hline 45 & 3 & 40950 & 15 & 330750 & 10 \\
\hline 60 & 4 & 46494 & 9 & 332640 & 20 \\
\hline 90 & 4 & 51408 & 12 & 464940 & 18 \\
\hline 420 & 7 & 55125 & 7 & 565488 & 22 \\
\hline 630 & 7 & 64260 & 17 & 598500 & 19 \\
\hline 1512 & 6 & 66528 & 12 & 646425 & 13 \\
\hline 3780 & 9 & 81900 & 18 & 661500 & 12 \\
\hline 5460 & 13 & 87360 & 16 & 716625 & 13 \\
\hline 7560 & 10 & 95550 & 14 & 790398 & 17 \\
\hline 8190 & 13 & 143640 & 19 & 791700 & 29 \\
\hline 9100 & 10 & 163800 & 20 & 859950 & 18 \\
\hline 15925 & 7 & 172900 & 19 & 900900 & 33 \\
\hline 16632 & 11 & 185976 & 12 & 929880 & 20 \\
\hline
\end{tabular}

\section{REFERENCES}

1. M. Garcia, On numbers with integral harmonic mean, Amer. Math. Monthly 61 (1954), 89-96. MR 15, 506; 1140.

2. S. W. Golomb, Powerful numbers, Amer. Math. Monthly 77 (1970), 848-855. MR $42 \# 1780$.

3. R. Guy and V. Klee, Monthly research problems, 1969-71, Amer. Math. Monthly 78 (1971), 1113-1122.

4. G. H. Hardy and E. M. Wright, An introduction to the theory of numbers, 4th ed., Oxford Univ. Press, London, 1960.

5. H.-J. Kanold, Über das harmonis che Mittel der Teiler einer natürlichen Zahl, Math. Ann. 133 (1957), 371-374. MR 19, 635.

6. O. Ore, On the averages of the divisors of a number, Amer. Math. Monthly 55 (1948), 615-619. MR 10, 284. script).

7. C. Pomerance, On a problem of Ore: harmonic numbers (unpublished manu-

8. H. N. Shapiro, Note on a theorem of Dickson, Bull. Amer. Math. Soc. 55 (1949), 450-452. MR 10, 514.

9. M. V. Subbarao, Are there an infinity of unitary perfect numbers? Amer. Math. Monthly 77 (1970), 389-390.

10. M. V. Subbarao and L. J. Warren, Unitary perfect numbers, Canad. Math. Bull. 9 (1966), 147-153. MR 33 \#3994.

DEPARTMENT OF MATHEMATICS, TEMPLE UNIVERSITY, PHIL ADELPHIA, PENNSYLVANIA 19121

SCHOOL OF BUSINESS, TEMPLE UNIVERSITY, PHILADELPHIA, PENNSYLVANIA 